\title{
Tunable transmission and harmonic generation in nonlinear metamaterials
}

\author{
Ilya V. Shadrivov, ${ }^{1, a)}$ Alexander B. Kozyrev, ${ }^{2}$ Daniel W. van der Weide, ${ }^{2}$ and \\ Yuri S. Kivshar ${ }^{1}$ \\ ${ }^{1}$ Nonlinear Physics Center, Research School of Physical Sciences and Engineering, \\ Australian National University, Canberra, Australian Capital Territory 0200, Australia \\ ${ }^{2}$ Department of Electrical and Computer Engineering, University of Wisconsin-Madison, \\ Madison, Wisconsin 53706, USA
}

(Received 1 September 2008; accepted 22 September 2008; published online 21 October 2008)

\begin{abstract}
We study the properties of a tunable nonlinear metamaterial operating at microwave frequencies. We fabricate the nonlinear metamaterial composed of double split-ring resonators and wires where a varactor diode is introduced into each resonator so that the magnetic resonance can be tuned dynamically by varying the input power. We show that at higher powers the transmission of the metamaterial becomes power dependent, and we demonstrate experimentally power-dependent transmission properties and selective generation of higher harmonics. (C) 2008 American Institute of Physics. [DOI: 10.1063/1.2999634]
\end{abstract}

Engineered microstructured metamaterials demonstrate many intriguing properties for the propagation of electromagnetic (em) waves such as negative refraction. Such materials have been studied extensively during recent years. ${ }^{1}$ Typically, such metamaterials are fabricated as composite structures created by many identical resonant scattering elements with size much smaller than the wavelength of the propagating em waves; such microstructured materials can be described in terms of macroscopic quantities-electric permittivity $\epsilon$ and magnetic permeability $\mu$. By designing the individual unit cell of metamaterials, one may construct composites with effective properties not occurring in nature.

Split-ring resonators (SRRs) are the key building blocks for the composite metamaterials, in particular, the materials having a negative refractive index. ${ }^{2}$ Recent theoretical studies have demonstrated how to dynamically tune or modulate the em properties of metamaterials ${ }^{3-8}$ and the fabrication of nonlinear SRRs has been demonstrated by placing a varactor $\operatorname{diode}^{9}$ or a photosensitive semiconductor ${ }^{10}$ within the gap of the resonator. The diode allows the SRR element to be tuned by an applied dc voltage or by a high-power signal as was shown already in experiment. ${ }^{9,11}$ These recent advances open a way for both fabrication and systematic study of nonlinear tunable metamaterials which may change their properties such as the transmission characteristics by varying the amplitude of the input em field.

It was shown theoretically that nonlinear metamaterials can demonstrate many intriguing features such as unconventional bistability, ${ }^{3,12}$ backward phase matching and harmonic generation, ${ }^{13-15}$ as well as parametric shielding of em fields. ${ }^{16}$ Some of these features have already been experimentally observed in nonlinear left-handed transmission lines which are model systems allowing for combining nonlinearity and anomalous dispersion. ${ }^{17-19}$ Moreover, first results on harmonic generation in infrared were reported in Ref. 20. Importantly, in such composite structures the microscopic electric fields can become much higher than the macroscopic electric field carried by the propagating em waves. This provides a simple physical mechanism for enhancing

${ }^{a)}$ Electronic mail: ivs124@rsphysse.anu.edu.au. nonlinear effects in the resonant structure with the lefthanded properties. Moreover, a very attractive goal is to create tunable metamaterial structures where the field intensity changes the properties of a composite structure, enhancing or suppressing the wave transmission.

In this letter, we report on the fabrication and experimental studies of the properties of the first nonlinear tunable metamaterial operating at microwave frequencies. Such a metamaterial was fabricated by modifying the properties of SRRs and introducing varactor diodes in each SRR element of the composite structure, ${ }^{9,11}$ such that the whole structure becomes dynamically tunable by varying the amplitude of the propagating em waves. In particular, we demonstrate the power-dependent transmission of the left-handed metamaterial (LHMS) and magnetic metamaterials (MMM) at higher powers, as was suggested earlier theoretically, ${ }^{3}$ and selective generation of higher harmonics.

A metamaterial sample (see Fig. 1) is fabricated from $0.5 \mathrm{~mm}$ thick Rogers R4003 printed circuit boards with a nominal dielectric constant of 3.4. Our design allows for reconfiguring metamaterial structures by rearranging the same boards. In particular, we make three sets of dielectric boards with the appropriate slot allocations: (i) without any metallization, (ii) with tin coated copper nonlinear SRRs, and (iii) with tin coated copper wires. Combining these

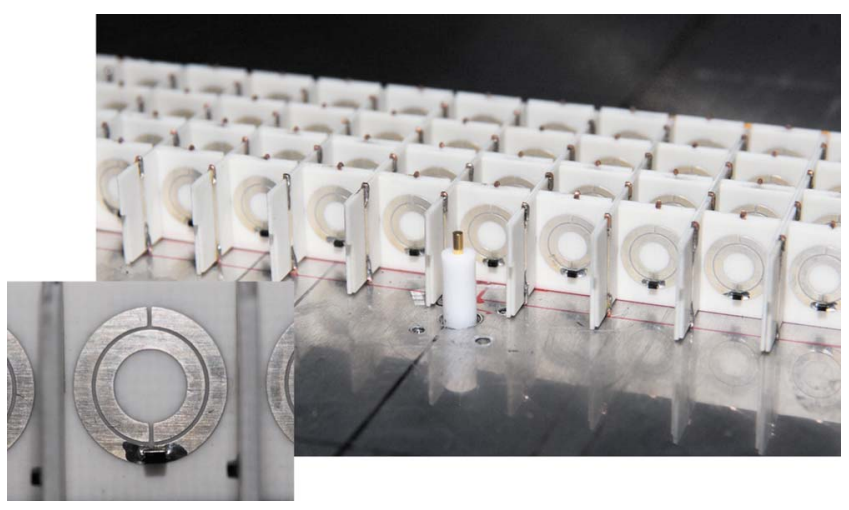

FIG. 1. (Color online) Photograph of the nonlinear tunable metamaterial created by square arrays of wires and nonlinear SRRs. Each SRR contains a varactor (see the inset) which provides power-dependent nonlinear response. 
boards in different pairs, we can achieve different types of composite media, including a wire medium, nonlinear MMM, and nonlinear LHM. A photograph of one of the nonlinear LHM structures is shown in Fig. 1. Each SRR contains a variable capacity diode (model Skyworks SMV1405) which introduces nonlinear current-voltage dependence and resulting nonlinear magnetic dipole moment to each SRR. ${ }^{11}$ In terms of effective medium parameters, the manufactured structure has nonlinear magnetization and nonlinear effective magnetic permittivity. ${ }^{3}$ Arrays of SRRs and wires form a square lattice with $29 \times 4 \times 1$ unit cells of the size of $10.5 \mathrm{~mm}$.

First, to identify the effect of the nonlinearity we measure the transmission properties of this tunable metamaterial for different values of the input power. To measure the em field scattering on our samples, the metamaterial slab is placed in a parallel plate waveguide. The planes of SRRs are aligned perpendicular to the parallel plate surfaces. The input antenna is placed at the midpoint of the lower plate, $2 \mathrm{~mm}$ from the metamaterial slab, in front of the central unit cell, and it consists of a Teflon-coated conductor of $1.26 \mathrm{~mm} \mathrm{di}$ ameter and $11 \mathrm{~mm}$ length. The Teflon coating provides a better energy coupling into the waveguide for the wavelengths of interest. The antenna is positioned perpendicular to the bottom plate, so that the excited electric field is polarized perpendicular to the plane, and thus parallel to the wires. The magnetic field of the wave has mainly an in-plane component, effectively exciting the SRRs. Close positioning of the source antenna to the metamaterial was chosen in order to funnel high em power into the metamaterial sample to observe nonlinear effects. We note that different positionings of the source antenna with respect to the central unit cell of the metamaterial gives slightly different quantitative results for the measured transmission; however, qualitatively all the results are identical. This effect appears due to different antenna impedance matchings to the sample. An identical antenna is placed in the center of the top plate and is used as a receiver for spectra measurements and for raster scan of the electric field distribution in the horizontal plane. The input antenna is excited using an Agilent E8364A vector network analyzer, the output of which is amplified by a HP 83020A $38 \mathrm{~dB}$ amplifier. For the transmission measurements, the receiving antenna is located $2 \mathrm{~cm}$ behind the metamaterial slab, in front of the central unit cell of the metamaterial, and it is connected to the network analyzer as well. The measurements of the electric field inside the waveguide are evaluated in terms of the magnitude and phase of the transmission coefficient $S_{21}$ between the input of the source and output of the receiver antenna. Due to the two-dimensional nature of the parallel plate waveguide, as well as symmetry of our sample, the electric field in the scanned area is expected to remain polarized mainly perpendicular to the plane of the plates.

Figure 2 shows the experimentally measured transmission coefficient as functions of the source power for different frequencies. The measurement is performed for the SRRwire structure. Due to the nonlinearity-induced shift of the resonant frequency of SRRs, studied earlier for a single $\mathrm{SRR},{ }^{11}$ the transmission of the metamaterial sample becomes dependent on the input power. In particular, depending on the value of the frequency, the metamaterial behaves as linear or strongly nonlinear, as shown in Fig. 2.

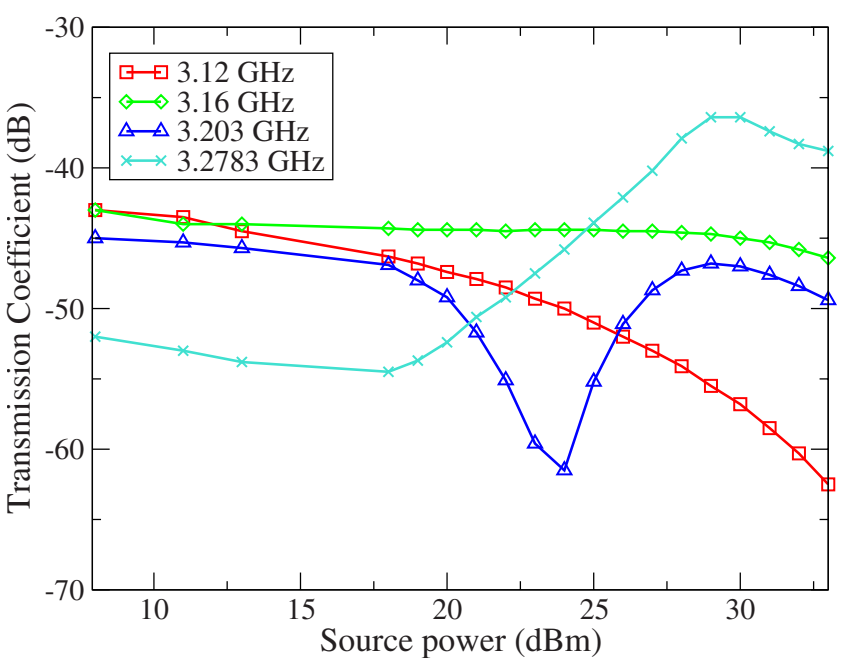

FIG. 2. (Color online) Experimentally measured transmission coefficient vs output power for different frequencies. Depending on the frequency, the metamaterial behaves as almost linear (green curve) or strongly nonlinear with different types of nonlinearity (three remaining curves).

In addition to the resonance shift measurements, we have studied harmonic generation in nonlinear LHM. In particular, we have measured the spectrum of the transmitted signal for different frequencies of the incident em wave. For this purpose, the input antenna was fed by the signal generated by on Agilent E4428C ESG vector signal generator and amplified by a $38 \mathrm{~dB}$ amplifier. The signal detected by the receiving antenna behind the metamaterial slab was analyzed using an Agilent E4448A PSA series spectrum analyzer. The location of the antennas was the same as in the previously described transmission coefficient measurements.

Figure 3 shows spectra of the signal detected by the receiving antenna behind the nonlinear LHM slab. Varying the input frequency, we observed efficient selective harmonic generation. Namely, second [Fig. 3(a)], third [Fig. 3(b)], and fourth [Fig. 3(c)] harmonics were selectively generated. Moreover, the generation of a comblike signal was also observed [Fig. 3(d)].

Selective generation of higher harmonics observed in our experiments is related to the transmission properties of the metamaterial. A particular harmonics dominates over fundamental harmonic and the other higher harmonics when its frequency corresponds to the transparency band. Results of the transmission coefficient measurements performed on our nonlinear LHMs indicate a right-handed transparency band with a maximum transparency at around $7 \mathrm{GHz}$. This value agrees well with the values of the higher harmonics dominating in our measurements. Furthermore, the presence of very high order harmonics in the spectrum of the transmitted signal manifests strong nonlinearity inside the metamaterial which potentially may lead to significant enhancement in nonlinear processes in artificial metamaterials as compared to conventional materials.

In conclusion, we have fabricated and analyzed the first tunable nonlinear metamaterial operating at microwave frequencies. The microwave metamaterial is composed of SRRS and wires where each SRR has a varactor diode, and it can be tuned dynamically by varying the input power. We have shown that such nonlinear metamaterials demonstrate the power-dependent transmission and selective generation of higher harmonics. We believe that our experimental re- 

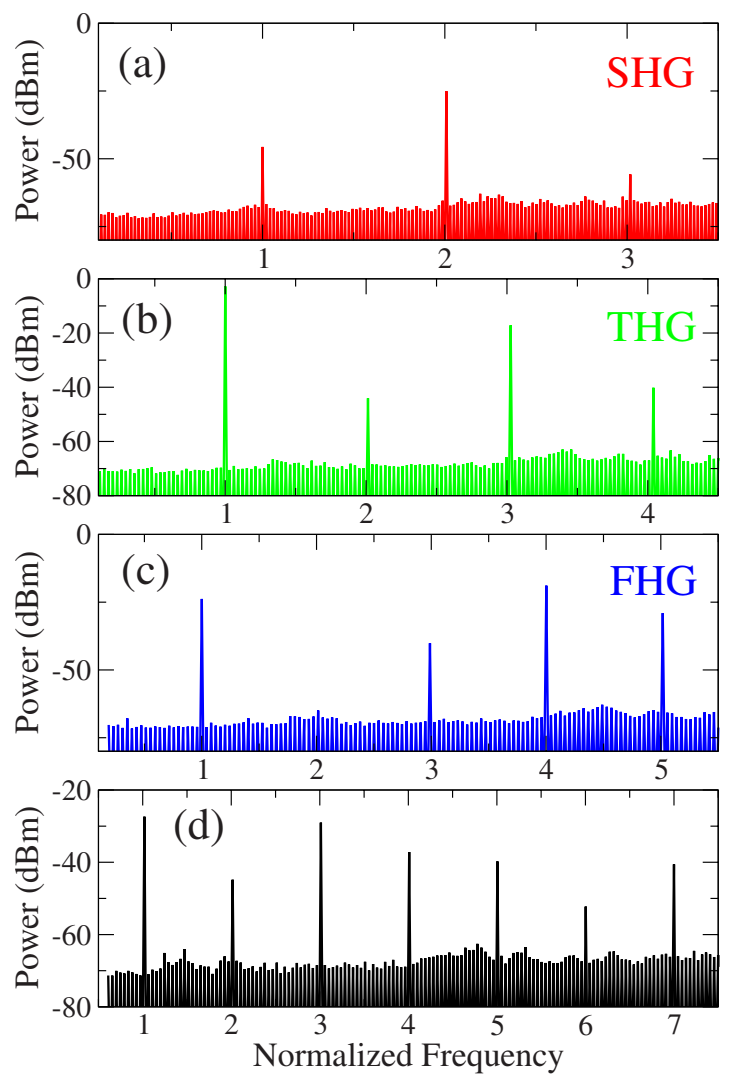

FIG. 3. (Color online) Spectra of the signals detected by the receiving antenna located behind the nonlinear LHM slab for different source frequencies: (a) $3.415 \mathrm{GHz}$, (b) $2.29 \mathrm{GHz}$, (c) $1.733 \mathrm{GHz}$, and (d) $1.668 \mathrm{GHz}$. The frequency on each graph is normalized to the corresponding source frequency. Power at the input antenna is $+30 \mathrm{dBm}$.

sults open a door for a systematic study of many intriguing features of nonlinear metamaterials earlier considered only theoretically for simplified models.

Two of the authors (I.S. and Y.K.) thank D. Powell, V. Shalaev, D. Smith, C. Soukoulis, and A. Zharov for useful discussions and suggestions. This work has been supported by the Australian Research Council through the Discovery projects, by the Australian Academy of Science through a travel grant, and by the Air Force Office of Scientific Research (AFOSR) through the MURI program (Grant No. F49620-03-1-0420).

${ }^{1}$ See, e.g., C. Soukoulis, Opt. Photonics News 17, 18 (2006), and references therein.

${ }^{2}$ D. Smith, W. Padilla, D. Vier, S. Nemat-Nasser, and S. Schultz, Phys. Rev. Lett. 84, 4184 (2000)

${ }^{3}$ A. A. Zharov, I. V. Shadrivov, and Yu. S. Kivshar, Phys. Rev. Lett. 91, 037401 (2003).

${ }^{4}$ M. Gorkunov and M. Lapine, Phys. Rev. B 70, 235109 (2004).

${ }^{5}$ M. Lapine, M. Gorkunov, and K. H. Ringhofer, Phys. Rev. E 67, 065601 (2003).

${ }^{6} \mathrm{~S}$. Lim, C. Caloz, and T. Itoh, IEEE Trans. Microwave Theory Tech. 52, 26782690 (2004).

${ }^{7}$ H.-T. Chen, W. J. Padilla, J. M. O. Zide, A. C. Gossard, A. J. Taylor, and R. D. Averitt, Nature (London) 444, 597 (2006)

${ }^{8}$ I. V. Shadrivov and Yu. S. Kivshar, in Physics of Negative Refraction and Negative Index Materials, edited by C. M. Krowne and Y. Zhang (Springer, Berlin, 2007), Chap. 12.

${ }^{9}$ I. V. Shadrivov, S. K. Morrison, and Yu. S. Kivshar, Opt. Express 14, 9344 (2006).

${ }^{10}$ A. Degiron, J. J. Mock, and D. R. Smith, Opt. Express 15, 1115 (2007).

${ }^{11}$ D. A. Powell, I. V. Shadrivov, Yu. S Kivshar, and M. V. Gorkunov, Appl. Phys. Lett. 91, 144107 (2007).

${ }^{12}$ M. W. Feise, I. V. Shadrivov, and Yu. S. Kivshar, Appl. Phys. Lett. 85, 1451 (2004)

${ }^{13}$ V. M. Agranovich, Y. R. Shen, R. H. Baughman, and A. A. Zakhidov, Phys. Rev. B 69, 165112 (2004).

${ }^{14}$ I. V. Shadrivov, A. A. Zharov, and Yu. S. Kivshar, J. Opt. Soc. Am. B 23, 529 (2006).

${ }^{15}$ A. K. Popov and V. Shalaev, Opt. Lett. 14, 2169 (2006).

${ }^{16}$ S. Feng and K. Halterman, Phys. Rev. Lett. 100, 063901 (2008).

${ }^{17}$ A. B. Kozyrev, H. Kim, A. Karbassi, and D. W. van der Weide, Appl. Phys. Lett. 87, 121109 (2005).

${ }^{18}$ A. B. Kozyrev, H. Kim, and D. W. van der Weide, Appl. Phys. Lett. 88, 264101 (2006).

${ }^{19}$ A. B. Kozyrev and D. W. van der Weide, Appl. Phys. Lett. 91, 254111 (2007).

${ }^{20}$ M. W. Klein, M. Wegener, N. Feth, and S. Linden, Opt. Express 15, 5238 (2007). 